\title{
Quantum Phase Engineering of Two-Dimensional Post- Transition Metals by substrates: Toward a Room-Temperature Quantum Anomalous Hall Insulator
}

\author{
Lizhi Zhang, ${ }^{1,2}$ Changwon Park, ${ }^{1,2}$ and Mina Yoon ${ }^{1,2, *}$ \\ ${ }^{1}$ Center for Nanophase Materials Sciences, Oak Ridge National Laboratory, Oak Ridge, \\ TN 37831, USA \\ ${ }^{2}$ Department of Physics and Astronomy, University of Tennessee, Knoxville, Tennessee \\ 37916, USA \\ *Email: myoon@ornl.gov
}

\section{Contents}

1. Adsorption energies of metal atoms, their bond lengths to the substrate, and the Young's modulus for $\mathrm{Ge}, \mathrm{Sn}$, and $\mathrm{Pb}$ on the graphane substrate.

2. Electronic properties and molecular dynamics simulation of $2 \sqrt{3} \times 2 \sqrt{3}$ Sn-graphane on $\mathrm{Cu}(111)$ surface.

3. The phonon-dispersion curves of $2 \sqrt{ } 3 \times 2 \sqrt{3}$ Sn-graphane system

4. Two different stable models for $3 \times 3$ Sn-graphane.

5. Electronic band structures and topological edge states of honeycomb $3 \times 3 \mathrm{Sn}$ graphane, $\sqrt{2} 1 \times \sqrt{ } 21$ Sn-graphane, $2 \sqrt{ } 3 \times 2 \sqrt{ } 3$ Ge-graphane, $2 \sqrt{3} \times 2 \sqrt{3}$ Pb-graphane, and $2 \sqrt{ } 3 \times 2 \sqrt{ } 3$ Ge/Sn-SiC(0001) systems.

6. Projected density of states of single Sn atom on graphane substrate.

7. Curie temperature based on Ising model.

8. Magnetic anisotropy of $2 \sqrt{ } 3 \times 2 \sqrt{ } 3 \mathrm{Ge}(\mathrm{Sn}$ and $\mathrm{Pb}$ )-graphane.

9. Comparison between first principles and tight-binding $p_{x y}$ band structures of $2 \sqrt{ } 3 \times 2 \sqrt{ } 3$ Sn-graphane.

10. The influence of the correlation energy $U$ to the SOC gaps and magnetic exchange energy 
1. Adsorption energies of metal atoms, their bond length to the substrate, and the Young's modulus for Ge, $\mathrm{Sn}$, and $\mathrm{Pb}$ on the graphane substrate.

TABLE S1. The M-C bond length L $(\AA)$, binding energy $E_{b}(\mathrm{eV})$, and biaxial modulus B (GPa) for $\mathrm{Ge}, \mathrm{Sn}$, and $\mathrm{Pb}$ on the graphane substrate. Here $E_{b}$ is defined as $E_{\mathrm{b}}=-\left(E_{\mathrm{tot}}-E_{\mathrm{sub}}-2 E_{\mathrm{atom}}\right) / 2$, where $E_{\text {tot }}$ is the total energy, $E_{\text {sub }}$ is the energy of $\mathrm{Hv}$-graphane, and $E_{\text {atom }}$ is the energy of single metal atoms.

\begin{tabular}{cccc}
\hline & $\mathrm{L}(\AA)$ & $\mathrm{E}_{\mathrm{b}}(\mathrm{eV})$ & $\mathrm{B}(\mathrm{GPa})$ \\
\hline $\mathrm{Ge}(3 \times 3)$ & 2.12 & 2.29 & 105.1 \\
$\mathrm{Ge}(2 \mathrm{~V} 3 \times 2 \sqrt{ } 3)$ & 2.11 & 2.08 & 130.5 \\
$\mathrm{Ge}(\mathrm{V} 21 \times \sqrt{ } 21)$ & 2.10 & 1.99 & 106.5 \\
$\mathrm{Sn}(3 \times 3)$ & 2.35 & 2.16 & 106.1 \\
$\mathrm{Sn}(2 \mathrm{~V} 3 \times 2 \mathrm{~V} 3)$ & 2.34 & 1.89 & 130.4 \\
$\mathrm{Sn}(\mathrm{V} 21 \times \sqrt{ } 21)$ & 2.32 & 1.71 & 106.9 \\
$\mathrm{~Pb}(3 \times 3)$ & 2.46 & 2.03 & 106.7 \\
$\mathrm{~Pb}(2 \mathrm{~V} 3 \times 2 \sqrt{ } 3)$ & 2.45 & 1.76 & 106.6 \\
$\mathrm{~Pb}(\mathrm{~V} 21 \times \sqrt{ } 21)$ & 2.43 & 1.54 & 131.1 \\
\hline \hline
\end{tabular}

\section{Electronic properties and molecular dynamics simulation of $2 \sqrt{3} \times 2 \sqrt{3}$ Sn- graphane on $\mathrm{Cu}(111)$ surface.}

For graphane with hydrogen vacancies synthesized on a $\mathrm{Cu}(111)$ surface [S1]: The $\mathrm{Cu}(111)$ substrate will not influence the electronic properties of Sn film (Figure S1). We perform ab initio MD simulations using a supercell of $2 \times 2$ unit cells of the $2 \sqrt{3} \times 2 \sqrt{3}$ model on the $\mathrm{Cu}(111)$ surface at $300 \mathrm{~K}$ up to $8 \mathrm{pm}$ and confirm that the structure is stable.
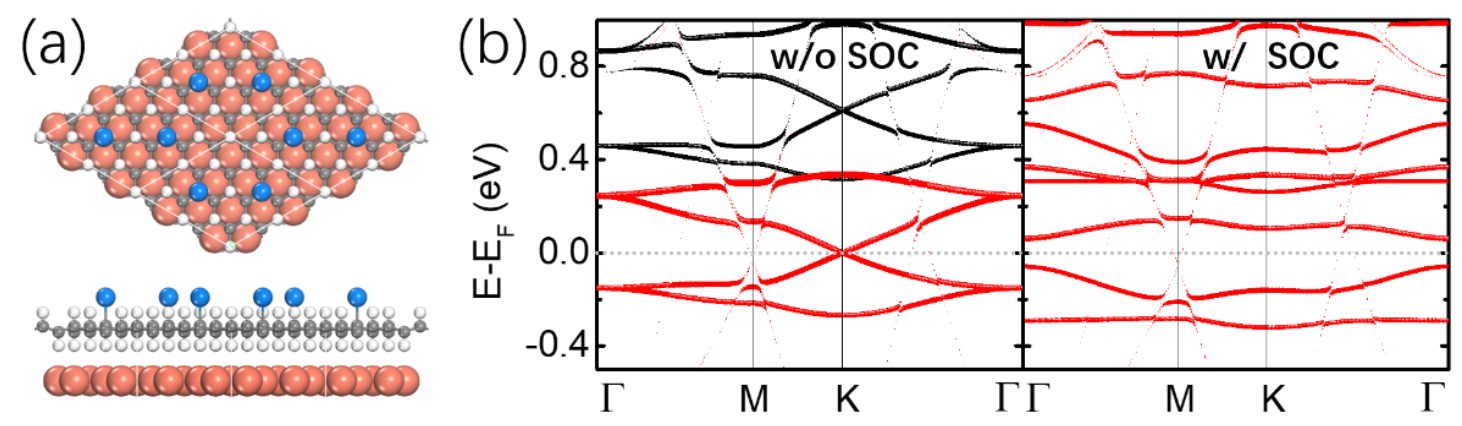

FIGURE S1. (a) Top- and side-view schematics of the atomic configuration of $2 \sqrt{ } 3 \times 2 \sqrt{ } 3$ Ge-graphane on $\mathrm{Cu}(111)$ surface. (b) Project band structure on Sn atoms without considering SOC. (c) Project band structure on Sn atoms within considering SOC. 
(a)
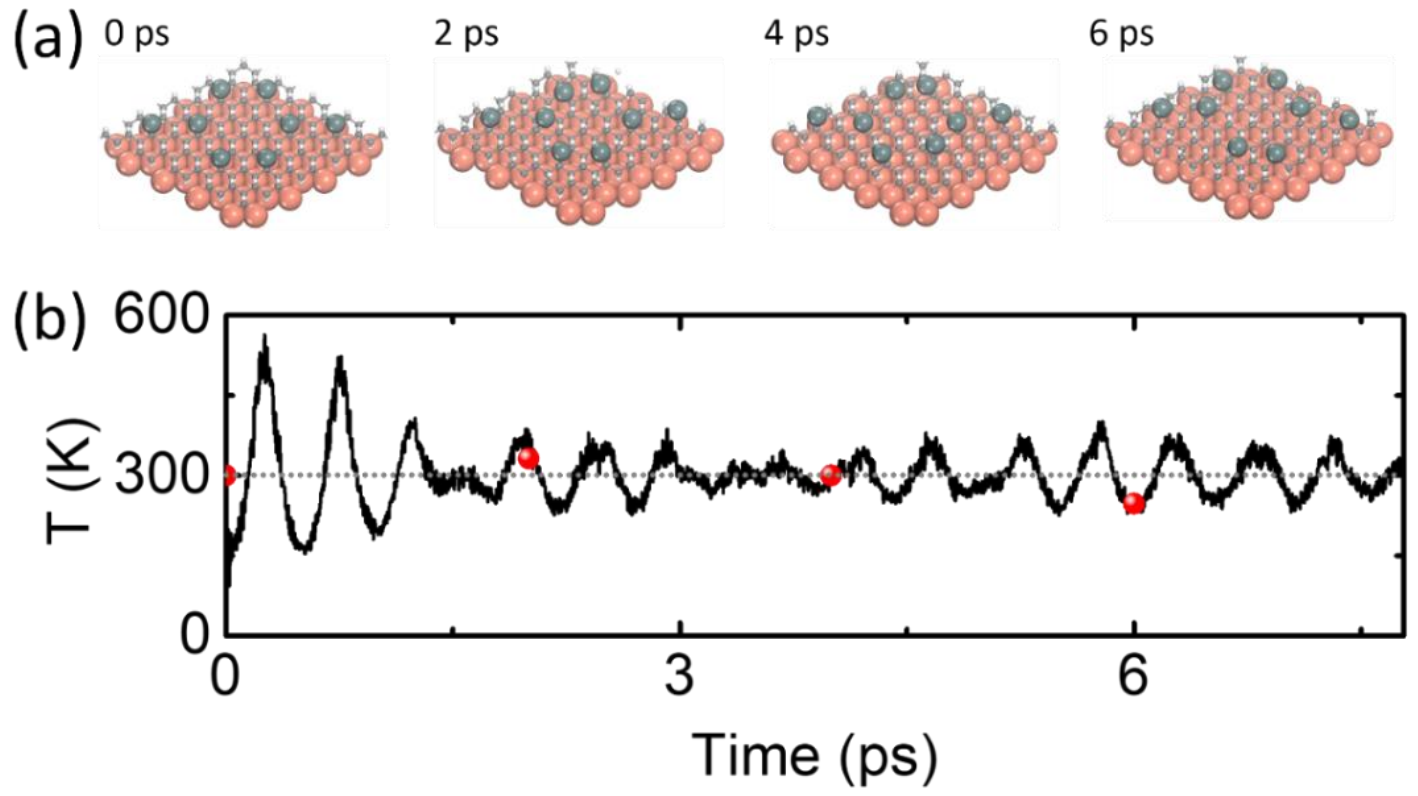

FIGURE S2. Molecular dynamics simulation of $2 \sqrt{3} \times 2 \sqrt{3}$ Sn-graphane on $\mathrm{Cu}$ (111) surface. (a) Snapshots at different times. (b) Temperature variation with time, and the red dots denote the times of snapshots taken in (a).

3. Two different stable models for the $2 \sqrt{ } 3 \times 2 \sqrt{ } 3$ Sn-graphane system.

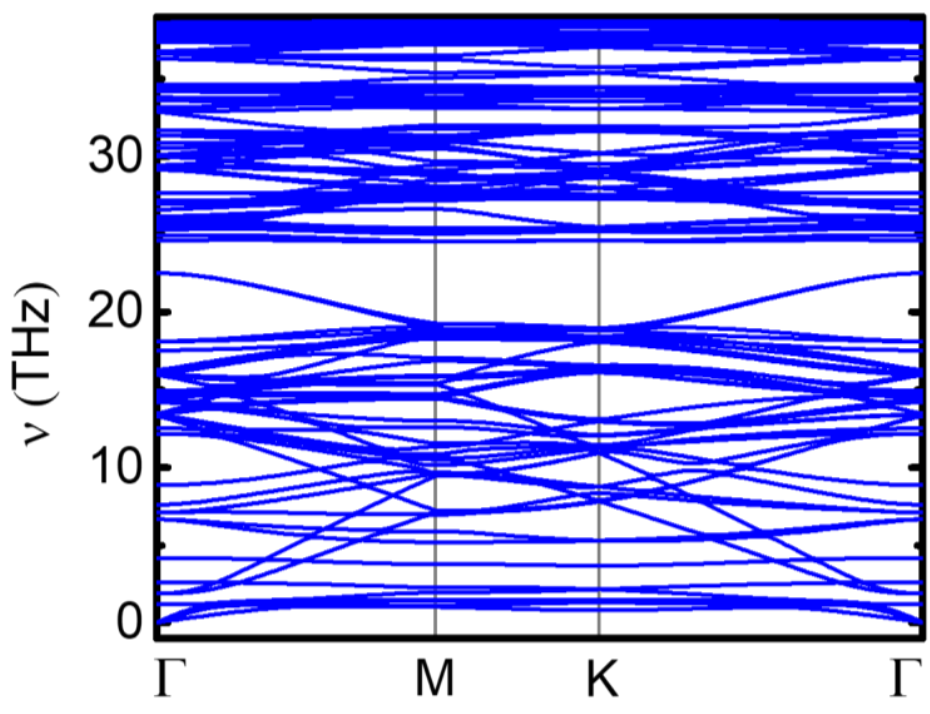

FIGURE S3. The phonon-dispersion curves of the $\mathrm{Sn}-2 \sqrt{3} \times 2 \sqrt{3}$ graphane system.

\section{Two different stable models for $3 \times 3$ Sn-graphane.}

For a $3 \times 3$ Sn-graphane system, there are two stable configurations, the diatomic model and the honeycomb model. The diatomic model is more stable, and the total energy difference between 
the two configurations is about $4.8 \mathrm{meV} / \mathrm{Sn}$. For the diatomic model, the distance between the two nearest Sn atoms is about $3.59 \AA$, which is smaller than that in the honeycomb model (4.39 $\AA$ ). Owing to the interaction between the Sn atoms, its magnetic moment is quenched, and the diatomic $3 \times 3 \mathrm{Sn}$-graphane is a semiconductor. The honeycomb model is a QAHI (Figure S6), where the SOC gap is about $81.8 \mathrm{meV}$, and the exchange energy is about $65.3 \mathrm{meV}$.

(a) diatomic

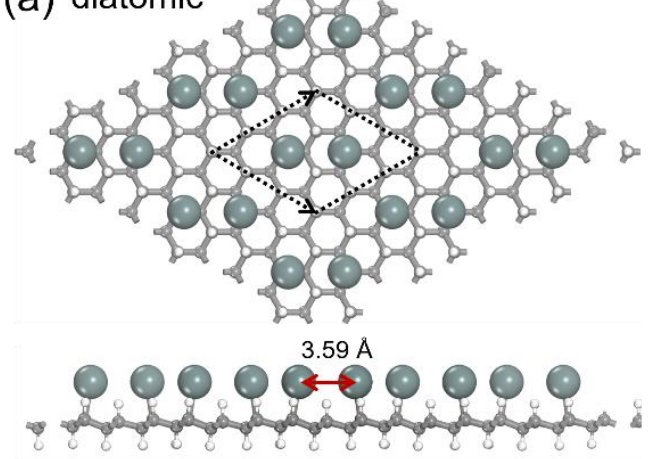

(b) honeycomb.
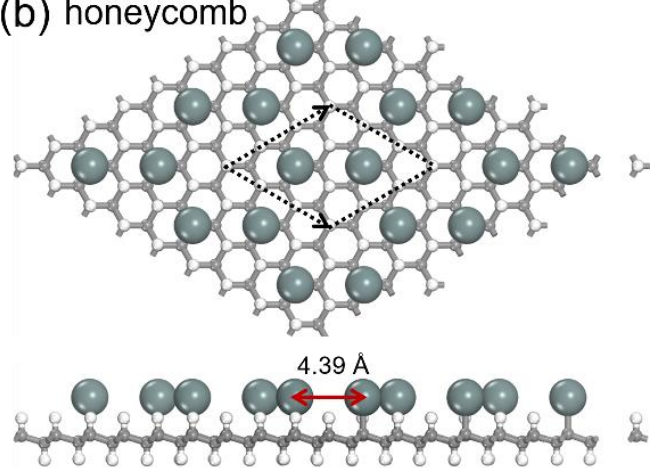

FIGURE S4. Top and side views of the schematic configurations of diatomic Sn (a) and honeycomb Sn (b) on $3 \times 3$ graphane substrates.
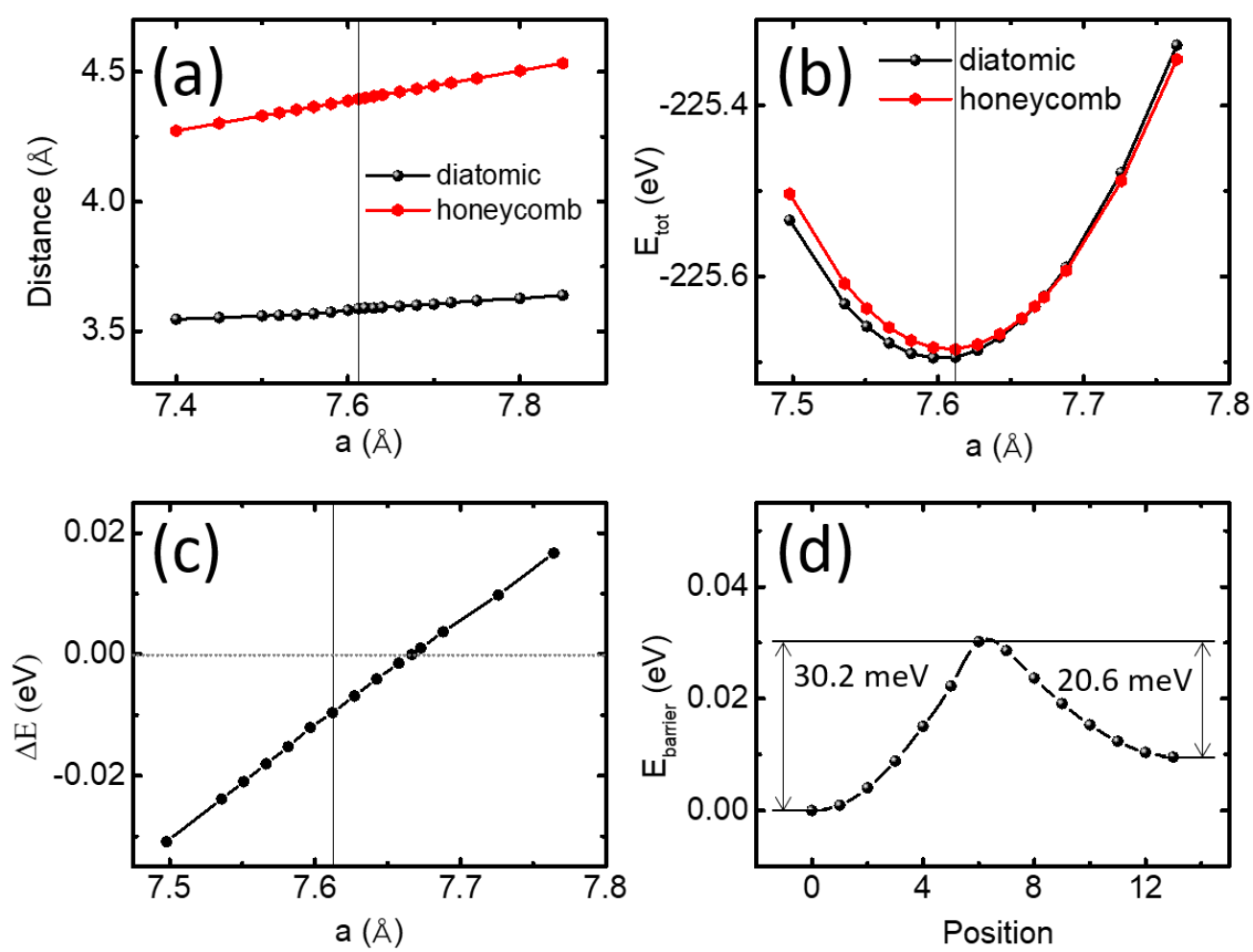

FIGURE S5. Sn-Sn distance (a), total energy (b) and total energy difference per unit cell (c) of honeycomb and diatomic model vs. the lattice constant around 7.612 A. (d) Energy barrier of two different models at $7.612 \AA$. 


\section{Electronic band structures and topological edge states of honeycomb $3 \times 3 \mathrm{Sn}$ - graphane, $\sqrt{2} 1 \times \sqrt{ } 21$ Sn-graphane, $2 \sqrt{ } 3 \times 2 \sqrt{3}$ Ge-graphane, $2 \sqrt{3} \times 2 \sqrt{3}$ Pb-graphane, and $2 \sqrt{ } \times 2 \times 2 \sqrt{3}$ Ge/Sn-SiC(0001).}

Similar to $2 \sqrt{ } 3 \times 2 \sqrt{3}$ Sn-graphane, honeycomb $3 \times 3$ Sn-graphane, $2 \sqrt{3} \times 2 \sqrt{3}$ Ge-graphane and $2 \sqrt{3} \times 2 \sqrt{3}$ Ge-SiC$(0001)$ are also QAHIs. For $\sqrt{2} 1 \times \sqrt{2} 1$ Sn-graphane, $2 \sqrt{ } 3 \times 2 \sqrt{3}$ Pb-graphane and $2 \sqrt{3} \times 2 \sqrt{3} \mathrm{Sn}-\mathrm{SiC}(0001)$, without considering SOC, the band structures are similar to that of $2 \sqrt{ } 3 \times 2 \sqrt{3}$ Sn-graphane, which indicate that they are half-semi-metal. Within considering SOC, topologically trivial gaps are opened at the Dirac point because of the excessively large SOC strength. There are no quantum Hall conductance around the Fermi level in the SOC gaps and no nontrivial topological edge states that connect the bulk states. Therefore, they are FMSC.

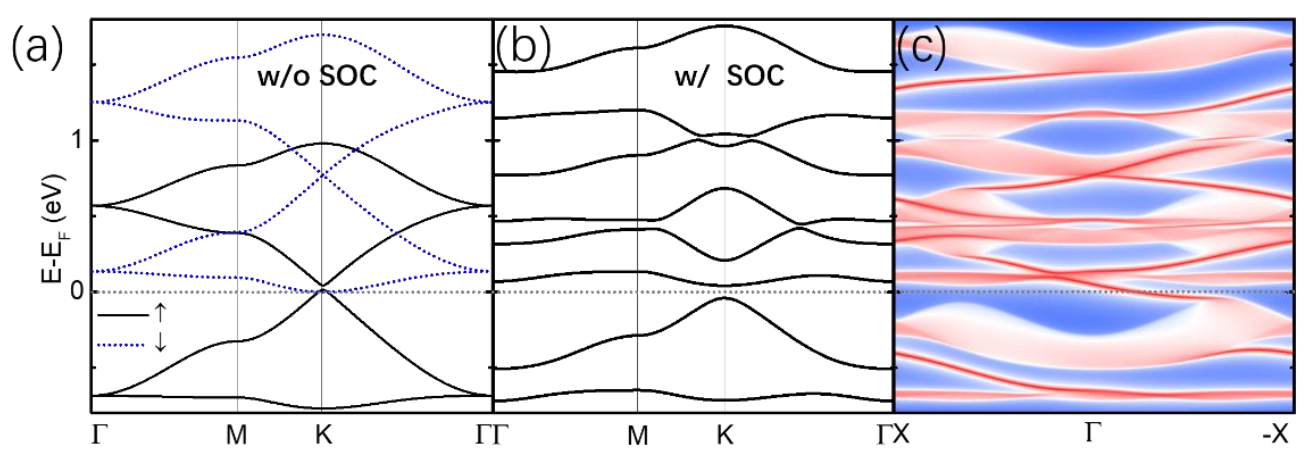

FIGURE S6. Electronic band structures and topological edge states of $\mathrm{Sn}$ on $3 \times 3$ graphane substrate (honeycomb model). (a) Band structures without and (b) within considering SOC, where the top inset indicates the atomic configuration. (c) The semi-infinite Dirac edge states around the Fermi level, where the shaded region indicates the bulk continuum states and the red lines show the edge states localized near the edge.

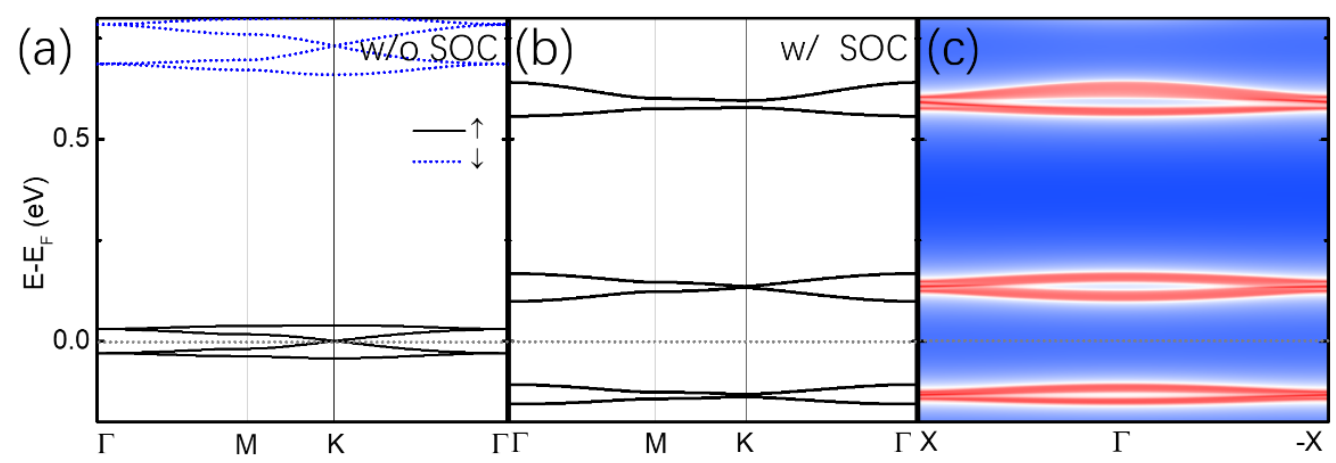

FIGURE S7. Electronic band structures and topological edge states of Sn on $\sqrt{ } 21 \times \sqrt{ } 21$ graphane substrate. (a) Band structures without and (b) within considering SOC, where the top inset indicates the atomic configuration. (c) The semi-infinite Dirac edge states around the Fermi level, where the shaded region indicates the bulk continuum states. 

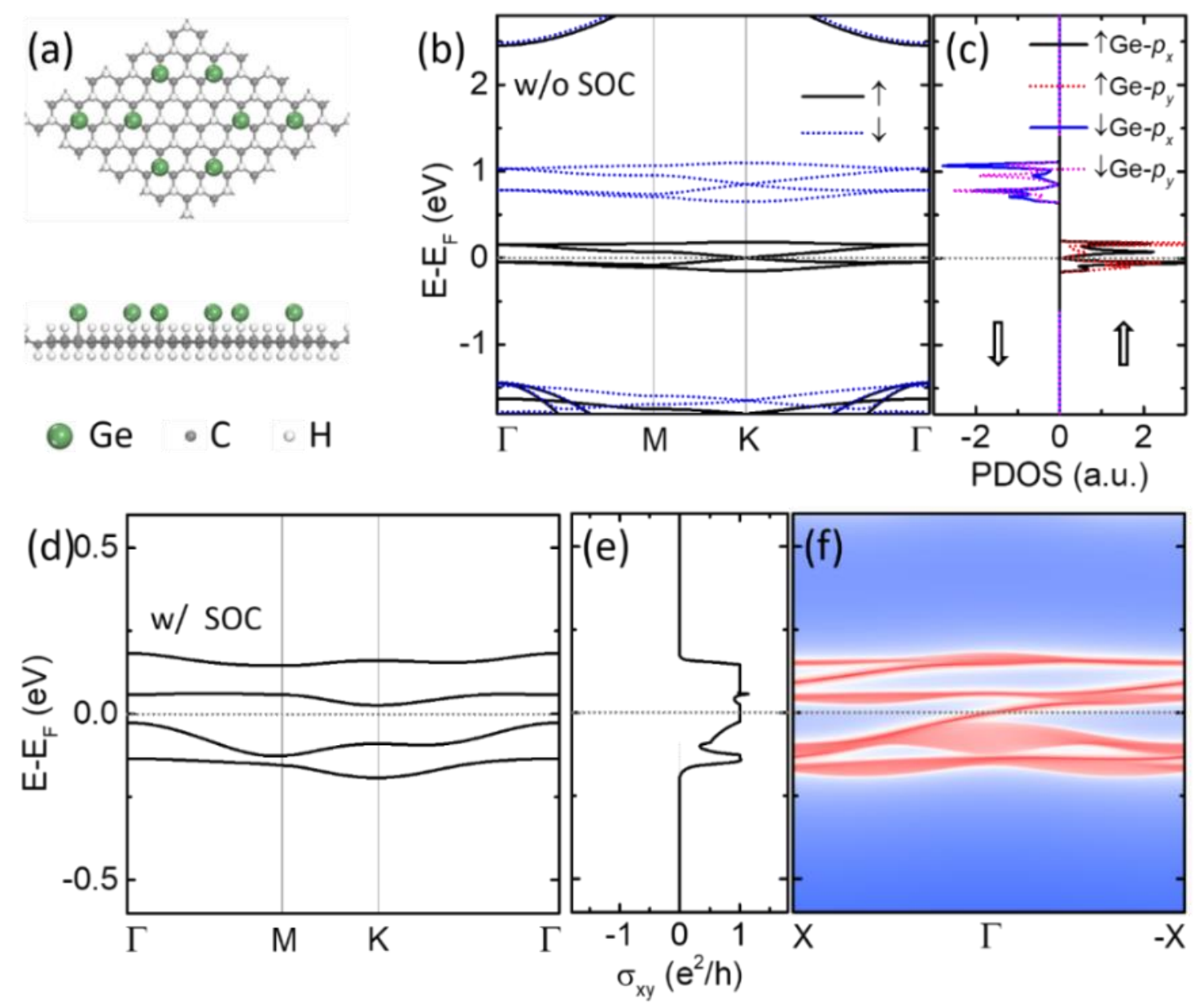

FIGURE S8. (a) Top- and side-view schematics of the atomic configuration of $2 \sqrt{ } 3 \times 2 \sqrt{ } 3$ Ge-graphane. (b) Band structure and (c) PDOS without considering SOC. (d) Band structure within considering SOC. (e) Quantum anomalous Hall conductance and (f) the semi-infinite Dirac edge states around the Fermi level, which indicate $2 \sqrt{3} \times 2 \sqrt{3}$ Ge-graphane is topologically nontrivial. The shaded region indicates the bulk continuum states, and the red lines show the edge states localized near the edge. 

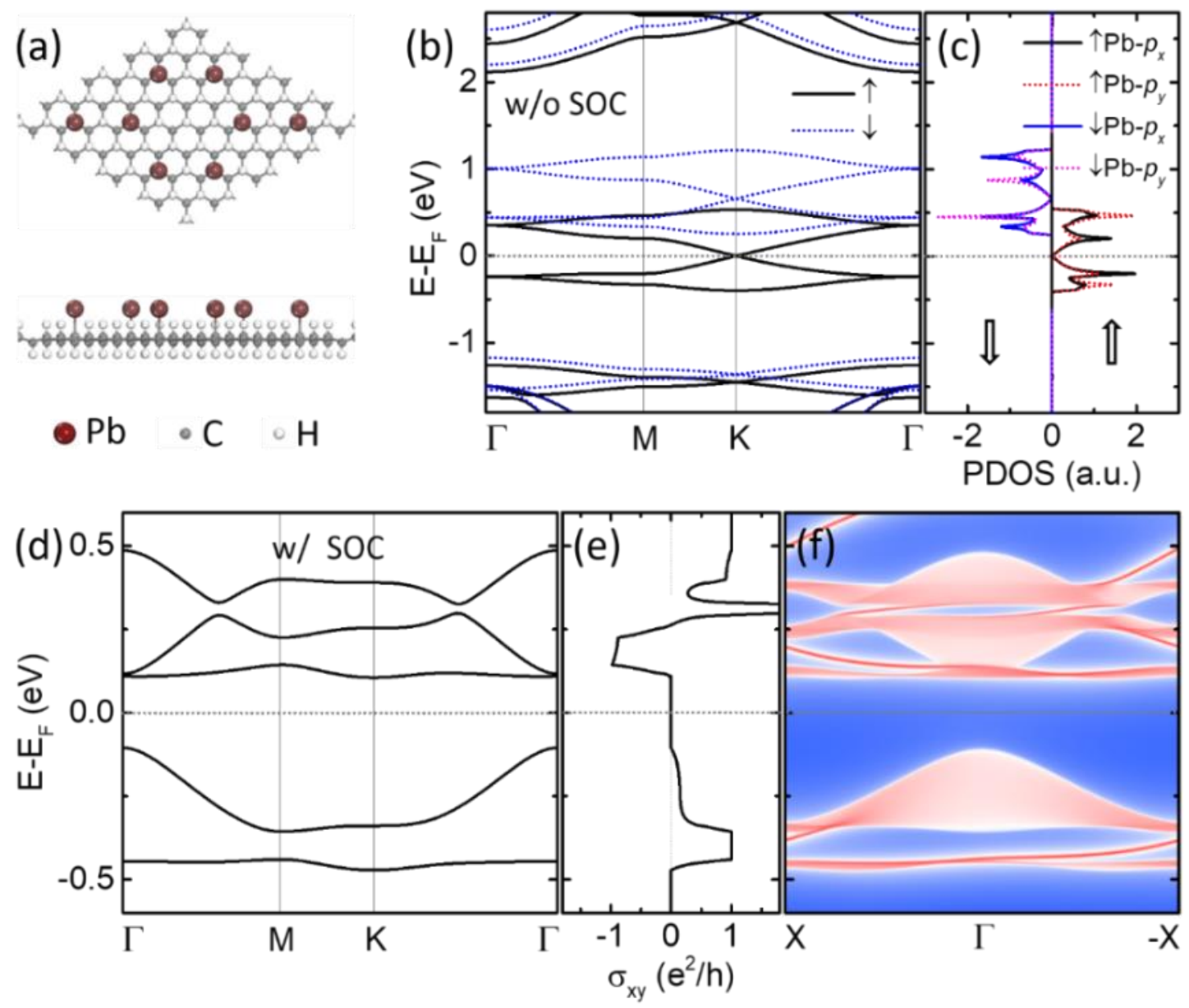

FIGURE S9. (a) Top- and side-view schematics of the atomic configuration of $2 \sqrt{3} \times 2 \sqrt{3} \mathrm{~Pb}$-graphane. (b) Band structure and (c) PDOS without considering SOC. (d) Band structure within considering SOC. (e) Quantum anomalous Hall conductance and (f) the semi-infinite Dirac edge states around the Fermi level, which indicate that $2 \sqrt{3} \times 2 \sqrt{3} \mathrm{~Pb}$-graphane is topologically trivial. The shaded region indicates the bulk continuum states. 


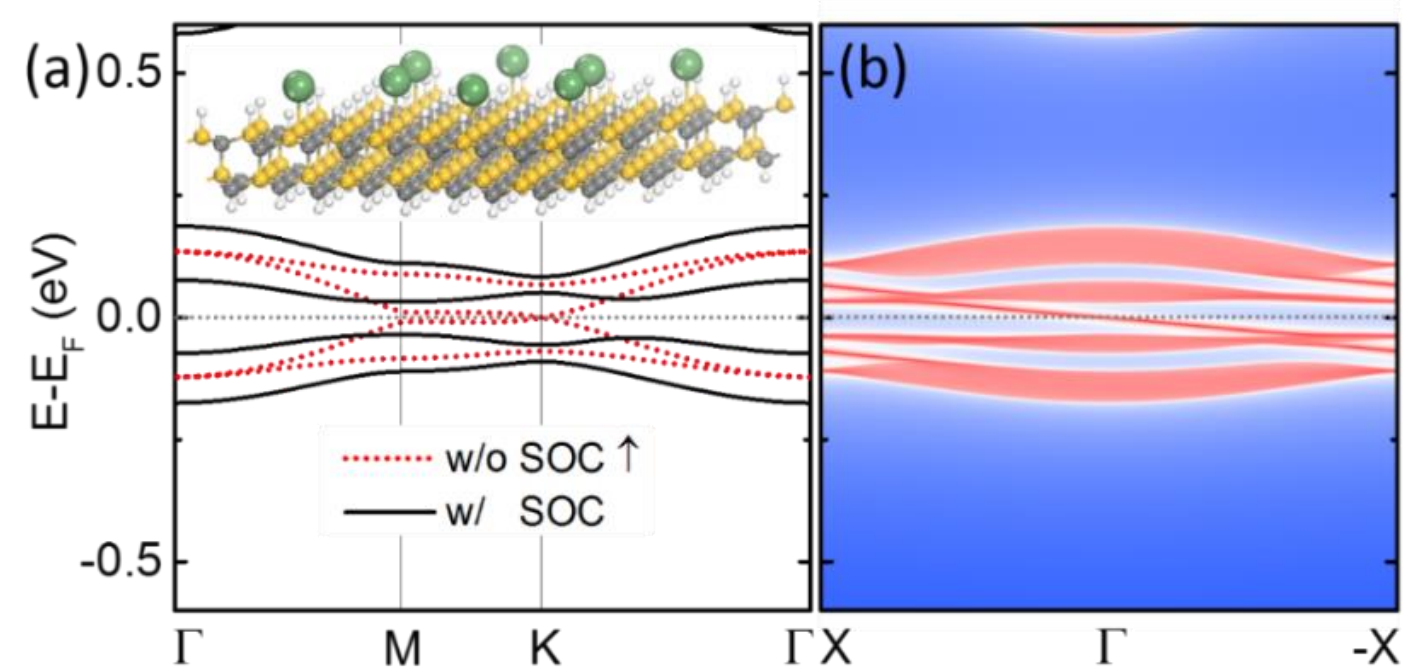

FIGURE S10. Electronic and topological properties of Ge on $2 \sqrt{3} \times 2 \sqrt{3} \mathrm{SiC}$ (111) substrate. (a) Band structures without and within considering SOC, where the top inset indicates the atomic configuration. (b) The semi-infinite Dirac edge states around the Fermi level, where the shaded region indicates the bulk continuum states and the red lines show the edge states localized near the edge.

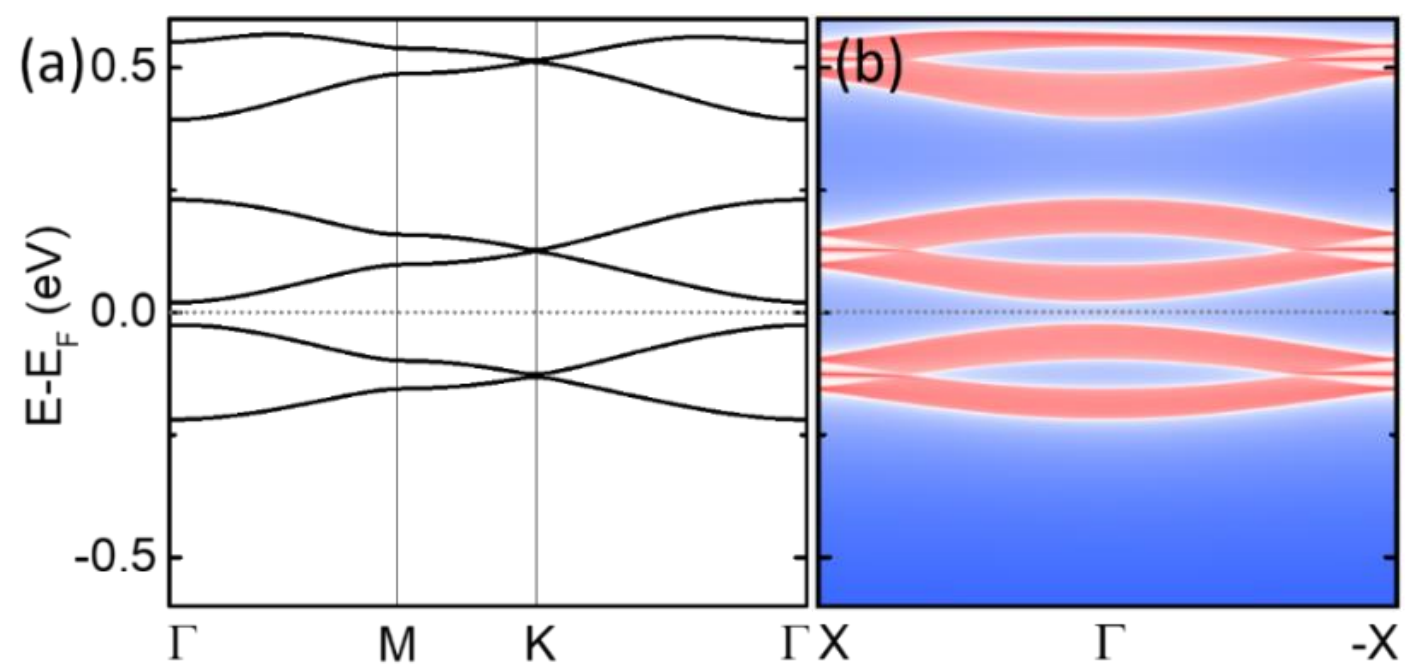

FIGURE S11. Electronic and topological properties of $\mathrm{Sn}$ on $2 \sqrt{3} \times 2 \sqrt{3} \mathrm{SiC}$ (111) substrate. (a) Band structures within considering SOC. (b) The semi-infinite Dirac edge states around the Fermi level, where the shaded region indicates the bulk continuum states. 


\section{Projected density of states (PDOS) of single Sn atom on graphane substrate.}

We construct a model containing one $\mathrm{Sn}$ atom and a $6 \sqrt{3} \times 6 \sqrt{3}$ graphane substrate. In this model, the distance between the two nearest Sn atoms is about $26.4 \AA$, and we can neglect the interaction between them.

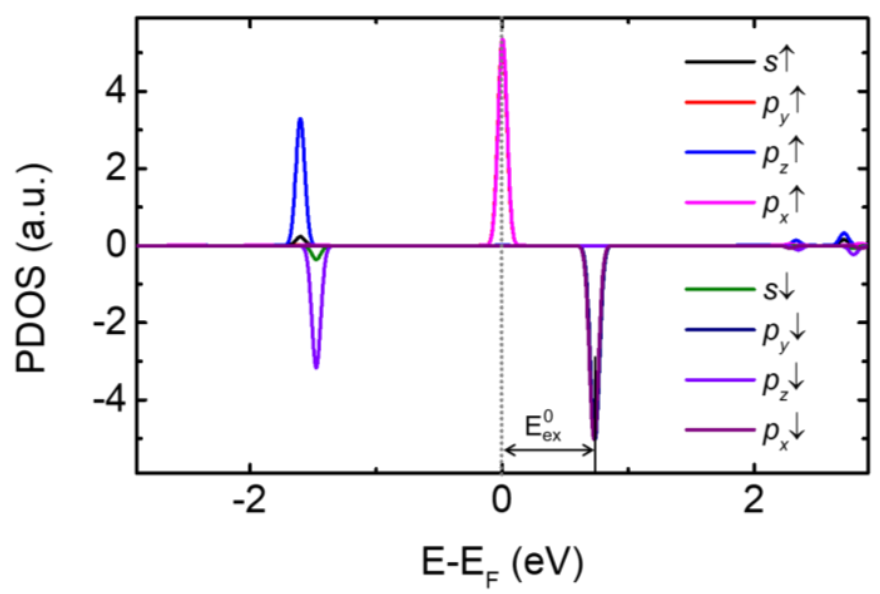

FIGURE S12. Projected density of states (PDOS) of single Sn atom on a graphane substrate, where $E_{e x}^{0}$ is defined as the exchange splitting of the $p_{x y}$ orbitals.

\section{Curie temperature based on Ising model.}

A Monte Carlo simulation is performed on a $1000 \times 1000$ 2D hexagonal lattice using an Ising Hamiltonian, $H=-\sum_{i, j} J S_{i} \cdot S_{j}$, where $J$ is the nearest-neighbor exchange coupling, $S$ is the net magnetic moment at the metal site, $i$ and $j$ stand for the nearest neighbors. We perform $10^{9}$ Monte Carlo steps for each temperature. We find that the calculated Curie temperature $T_{c}$ is about $680 \mathrm{~K}$.

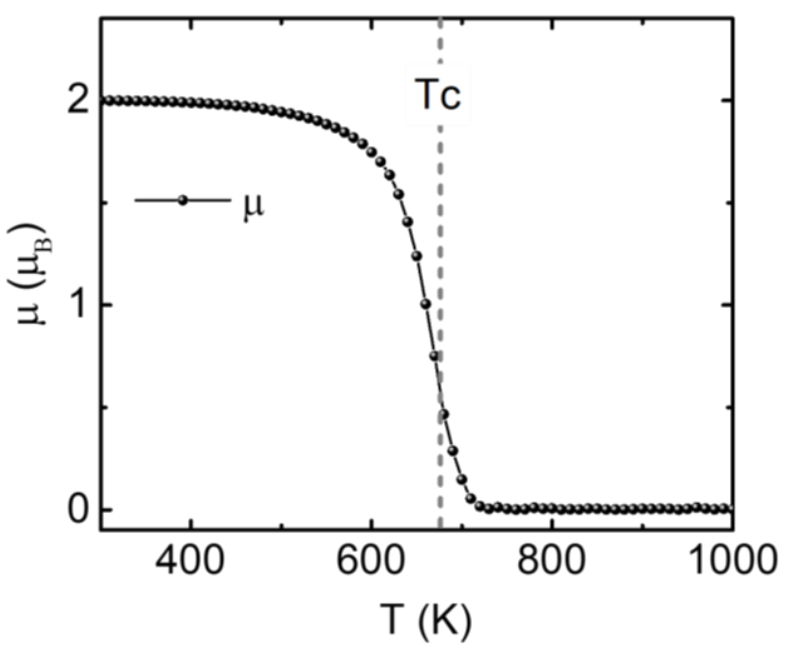

FIGURE S13. Variations of the average magnetic moment calculated for hexagonal $2 \sqrt{3} \times 2 \sqrt{3}$ Sn-graphane with respect to temperature $T$. 


\section{Magnetic anisotropy of $2 \sqrt{ } 3 \times 2 \sqrt{ } 3 \mathrm{Sn}$ (Ge and Pb)-graphane.}
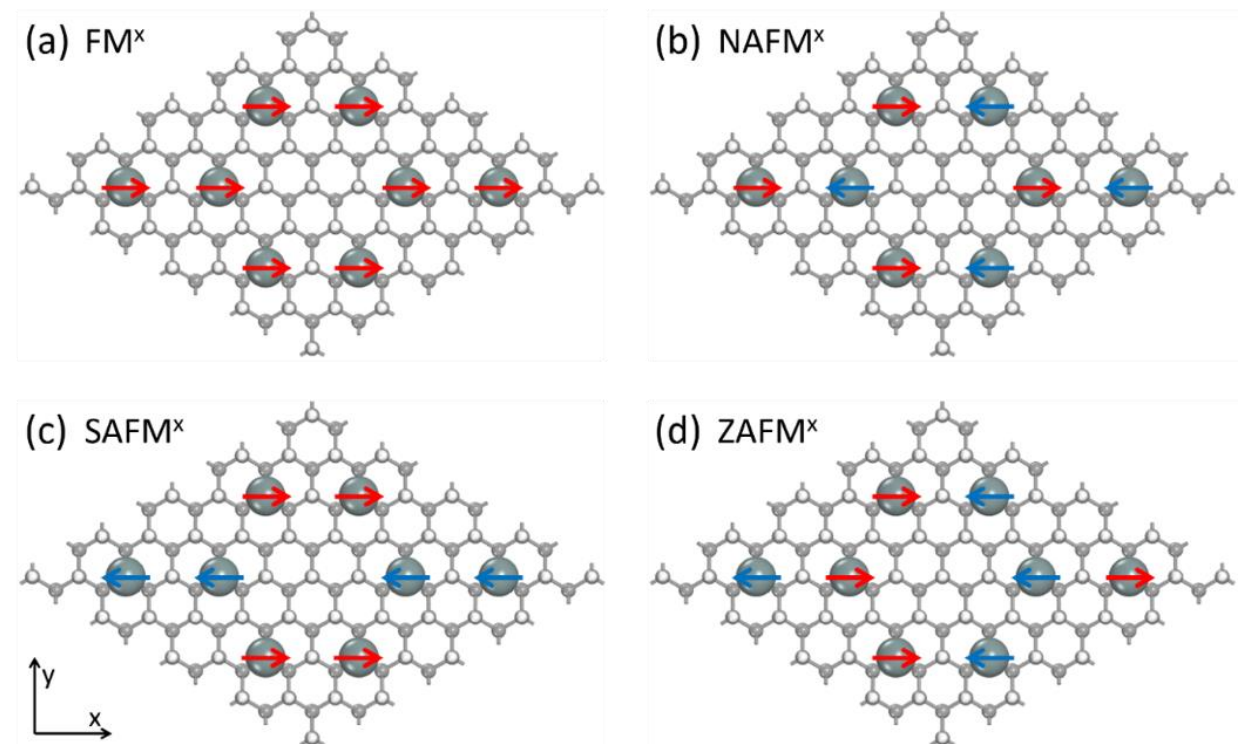

FIGURE S14. Possible configurations of spins on the honeycomb lattice: (a) ferromagnet (FM), (b) Néel antiferromagnet (NAFM), (c) stripy AFM (SAFM), and (d) zigzag AFM (ZAFM).

TABLE S2. The total energy $E_{\text {tot }}$ difference per unit cell (in meV, relative to $E_{\text {tot }}$ of $\mathrm{FM}^{\mathrm{z}}$ ground state) for several magnetic configurations of $\mathrm{Ge}(\mathrm{Sn}$ and $\mathrm{Pb}$ ) atoms (shown in Figure $\mathrm{S} 8$ ) calculated by the GGA+SOC method.

\begin{tabular}{lccccccccc}
\hline \hline & FM $^{2}$ & NAFM $^{2}$ & SAFM $^{2}$ & ZAFM $^{2}$ & FM $^{x}$ & NAFM $^{x}$ & SAFM $^{x}$ & ZAFM $^{x}$ & FM $^{y}$ \\
\hline Ge-graphane & 0.0 & 83.6 & 45.7 & 12.9 & 21.2 & 100.3 & 88.5 & 41.9 & 21.1 \\
Sn-graphane & 0.0 & 148.4 & 68.6 & 30.9 & 27.2 & 142.3 & 135.4 & 68.4 & 27.1 \\
Pb-graphane & 0.0 & 142.5 & 3.2 & 0.9 & 103.8 & 105.0 & 153.6 & 125.6 & 103.7 \\
\hline \hline
\end{tabular}

Within the SOC, we calculate the total energy difference for different spin configurations on the honeycomb lattice, which are all shown in Table S2. According to our calculation, the outof-plane ferromagnetic states are the ground states. The magnetic anisotropy energy $E_{M A E}$ is defined as $E_{M A E}=E_{\|}-E_{\perp}$, where $E_{\|}$and $E_{\perp}$ represent the ground state energies of the system when the spin moment is parallel or perpendicular to the 2D plane. Then, we get $E_{M A E}=E_{F M, x}$, and the $E_{M A E}$ increases as the SOC strength increases (from Ge to $\mathrm{Pb}$ ). Based on the model Hamiltonian of the xxz Heisenberg model,

$$
H=-\frac{1}{2}\left(J \sum_{i, i^{\prime}} \vec{s}_{i} \cdot \vec{s}_{i^{\prime}}+\lambda \sum_{i, i^{\prime}} s_{i}^{Z} \cdot s_{i^{\prime}}^{Z}\right),
$$

where $J$ is the isotropic exchange coupling, $\lambda$ is the anisotropic exchange coupling, $i$ runs over the entire lattice of metal atoms, and $i^{\prime}$ corresponds to the nearest neighbors. The first term in 
the Hamiltonian describes the Heisenberg isotropic exchange and the second term is the anisotropic exchange. If $\mathrm{J}>0$, the $2 \mathrm{D}$ system favors ferromagnetic interactions, and if $\lambda=0$, the 2D system implies a completely isotropic exchange interaction. Based on DFT calculations, we have the total energy of the ground state for four ground states: (i) ferromagnetic out of plane (FM, z), (ii) antiferromagnetic out of plane (AFM, z), (iii) ferromagnetic in plane (FM, $\mathrm{x}$ ), and (iv) antiferromagnetic in plane (AFM, x). Based on the spin model, we get the relationship between $J, \lambda$, and these four ground states: [S2]

$$
\begin{gathered}
E_{A F M, x}-E_{F M, x}=6 S^{2} J, \\
E_{A F M, z}-E_{F M, z}=6 S^{2}(J+\lambda),
\end{gathered}
$$

with $\mathrm{S}=1 / 2$ for $\mathrm{Sn}(\mathrm{Ge}$ and $\mathrm{Pb})$.

\section{Comparison between first-principles and tight-binding $p_{x y}$ band structures of $2 \sqrt{3} \times 2 \sqrt{3}$ Sn-graphane.}

We can use the $p_{x y}$ four-band model to fit the band structures of $2 \sqrt{3} \times 2 \sqrt{3}$ Sn-graphane around the Fermi level. According to our fitting, we can obtain the parameters in the effective Hamiltonian: where the fitting parameters are $\lambda_{S O}=0.27, t_{p p \sigma}=0.196, t_{p p \pi}=-0.041$, and $E_{e x}=0.38$. We can find that the tight-binding model fits very well with the DFT results, which confirms that our model captures all the essential features of topological properties.

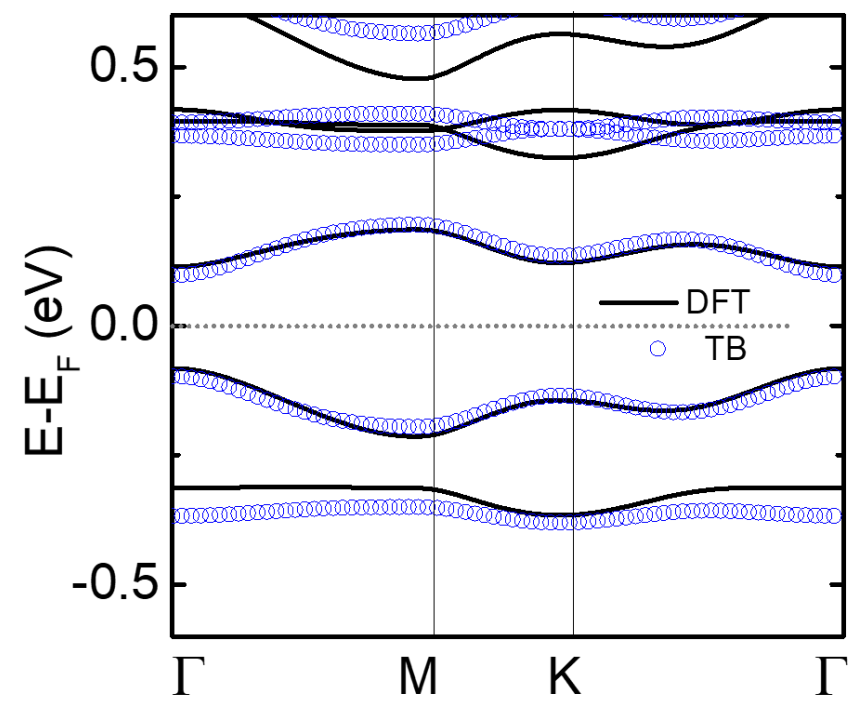

FIGURE S15. Comparison between first-principles and tight-binding $p_{x y}$ band structures of Sn-graphane, 


\section{The influence of the correlation energy $U$ to the SOC gaps and magnetic exchange energy}

We performed a new set of calculations to study the influence of the correlation energy $U$ on the electronic, magnetic, and topological properties. Our calculations show that the correlation affects only the splitting of $p$-orbitals around the Fermi level of Sn atoms (Here, there are no influences to the $d$-orbitals, because the bands around the Fermi level only come from the $p$ orbitals of Sn atoms.), which will increase the band gap and exchange energy of the system. Figure R1 shows that the spin-orbit coupling (SOC) gaps increase slightly with $U$, while the exchange energy decreases with $U$. We confirmed that the topological properties of the system are robust against $U$.

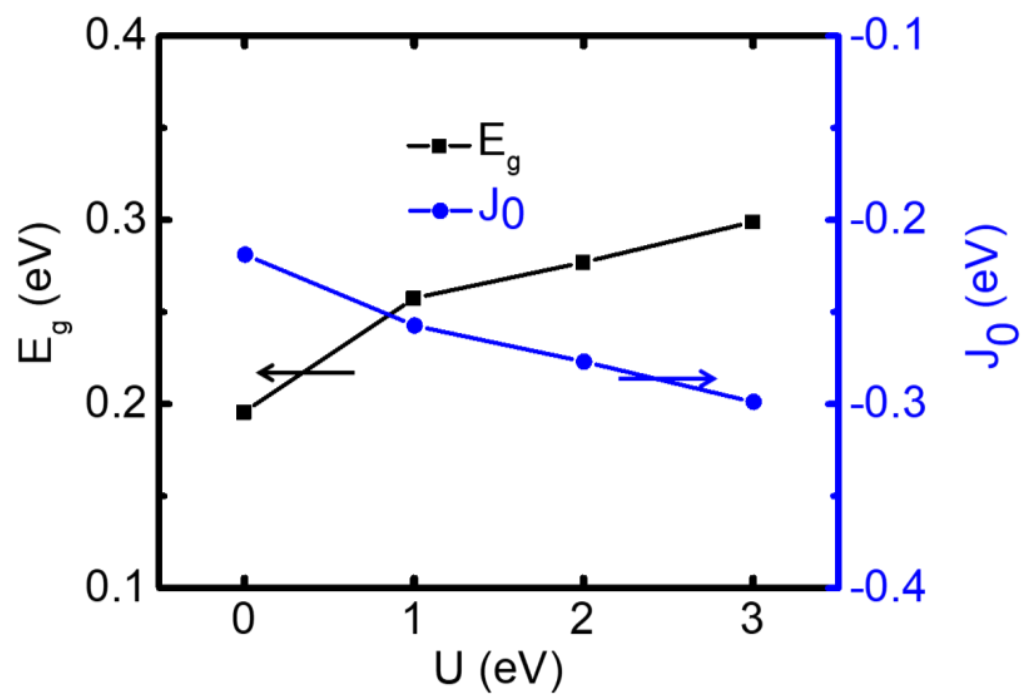

FIGURE S16. SOC energy gaps $\mathrm{E}_{\mathrm{g}}$ and magnetic exchange energy $\mathrm{J}_{0}$ vs the correlation energy $\mathrm{U}$.

\section{References}

[S1] C. Lin, Y. Feng, Y. Xiao, M. Dürr, X. Huang, X. Xu, R. Zhao, E. Wang, X. Li, and Z. Hu, Nano Lett. 15, 903 (2015).

[S2] J. L. Lado and J. Fernández-Rossier, 2D Mater. 4, 035002 (2017). 